\title{
COMPLETELY FREE LATTICES GENERATED BY PARTIALLY ORDERED SETS
}

\author{
BY \\ R. A. DEAN
}

1. Introduction. A description of the free lattice, $\mathrm{FL}(n)$, generated by $n$ unordered elements has been given by P. M. Whitman $[5 ; 6]$ for any cardinal, $n$. A description of a free lattice, $\operatorname{FL}(P)$, generated by any partially ordered set, $P$, has been given by $\mathrm{R}$. P. Dilworth [2]. This lattice has three chief properties: (1) $P$ is embedded $\left.{ }^{1}\right)$ in $\operatorname{FL}(P)$, (2) least upper and greatest lower bounds existing for pairs of elements in $P$ are preserved in $F L(P)$, and (3) any lattice providing a minimal embedding for $P$ with property (2) is a lattice homomorphic image of $\mathrm{FL}(P)$. In that same paper Dilworth called the completely free lattice, $\mathrm{CF}(P)$, generated by $P$, the lattice whose three chief properties are: $\left(1^{\prime}\right) P$ is embedded in $\mathrm{CF}(P),\left(2^{\prime}\right)$ the ordering of $P$ is preserved in $\mathrm{CF}(P)$, and $\left(3^{\prime}\right)$ any lattice providing a minimal embedding for $P$ with property $\left(2^{\prime}\right)$ is a lattice homomorphic image of $\mathrm{CF}(P)$. Thus in $\mathrm{CF}(P)$, the only least upper and greatest lower bounds which are preserved are those between comparable elements. The present paper investigates these completely free lattices. In $\S \S 2$ and 3 the techniques of Whitman $[5 ; 6]$ are applied to these lattices and many of his results are easily extended to this case. In particular, the word problem is solved in these lattices, a canonical form is shown to exist for each word, and necessary and sufficient conditions are given for a finite subset of $\mathrm{CF}(P)$, considered as a partially ordered set, to generate a completely free sublattice.

When $P$ has no bounds, other than between comparable elements, to be preserved, $\mathrm{CF}(P)$ and $\mathrm{FL}(P)$ are identical and in this event the solution to the word problem in $\mathrm{CF}(P)$ becomes very useful and provides a decision method easier to employ than that given by T. Evans [3]. Moreover the class of lattices for which the word problem is solved by the present method contains some lattices to which Evans' method is inapplicable. An example is the lattice $\mathrm{FL}(P)$ when $P$ consists of any number of disjoint infinite chains.

In $\$ 4$ two examples are given in which the decision method is particularly effective. The first partially ordered set considered is the set $P$ consisting of two disjoint chains of two elements each, $t>u$ and $v>w$. The second set $Q$ consists of two disjoint chains, one of four elements, $a>b>c>d$, and the other of a single element, e. $\mathrm{CF}(P)$ is identical with $\mathrm{FL}(P)$, and $\mathrm{CF}(Q)$ is identical with $\mathrm{FL}(Q)$. Sorkin [4] showed that these lattices contain chains

Presented to the Society, September 2, 1955; received by the editors November 14, 1955.

(1) For the definition of this term and others appearing in the introduction, see $\$ 2$. 
of infinite length. Here it is shown that $\mathrm{FL}(P)$ is a sublattice of $\mathrm{FL}(Q)$, and infinite chains are constructed in $\mathrm{FL}(P)$.

2. CF $(P)$. Let $P$ be a partially ordered set with elements $\left({ }^{2}\right) p_{i}$ and order relation $\geqq$. Words or lattice polynomials on the elements $p_{i}$ and their lengths are defined inductively by Definition 1 .

Definition 1. (i) For all $i, p_{i}$ is a word of length 1.

(ii) If $A$ and $B$ are words of length $\lambda(A)$ and $\lambda(B)$ respectively then $A \cup B$ and $A \cap B$ are words of length $\lambda(A)+\lambda(B)$.

Next the relation $(\geqq)$ is extended to the set of words formed from the $p_{i}$.

Definition 2. Let $A$ and $B$ be two words on the $p_{i}$. $A \geqq B$ if and only if one or more of the following hold $\left({ }^{3}\right)$ :

(i) $A \equiv p_{i}$ and $B \equiv p_{j}$ and $p_{i} \geqq p_{j}$ in $P$. Proceeding recursively:

(ii) (2.1) $A \equiv A_{1} \cup A_{2}$ and $A_{1} \geqq B$ or $A_{2} \geqq B$,

(2.2) $A \equiv A_{1} \cap A_{2}$ and $A_{1} \geqq B$ and $A_{2} \geqq B$,

(2.3) $B \equiv B_{1} \cup B_{2}$ and $A \geqq B_{1}$ and $A \geqq B_{2}$,

(2.4) $B \equiv B_{1} \cap B_{2}$ and $A \geqq B_{1}$ or $A \geqq B_{2}$.

Lemma 1. $A \geqq B_{1} \cup B_{2}$ implies $A \geqq B_{1}$ and $B_{2}$. Dually $A_{1} \cap A_{2} \geqq B$ implies $A_{1}$ and $A_{2} \geqq B$.

Proof. A proof is given for the first half of the lemma and the second half then follows by duality. The proof proceeds by induction on $\lambda(A)$. When $\lambda(A)=1, A \equiv p_{i}$, for some $i$. Thus $p_{i} \geqq B_{1} \cup B_{2}$ and from Definition 2 , the only applicable rule is (2.3). Assuming the result when $\lambda(A)<k$, let $\lambda(A)=k$.

Case 1. $A \equiv A_{1} \cup A_{2}$. From Definition 2, $A \geqq B_{1} \cup B_{2}$ implies either (2.1) or (2.3) holds. If (2.3) holds the result is true, thus assume that (2.1) holds, and without loss of generality, that $A_{1} \geqq B_{1} \cup B_{2}$. Then by induction $A_{1} \geqq B_{i}$, $i=1$ and 2 , and by (2.1) again $A_{1} \cup A_{2} \geqq B_{i}, i=1$ and 2 .

Case 2. $A \equiv A_{1} \cap A_{2}$. From Definition $2, A \geqq B_{1} \cup B_{2}$ implies either (2.2) or (2.3) holds. If (2.3) holds the result is true; thus assume that (2.2) holds. Hence for $i=1$ and $2, A_{i} \geqq B_{1} \cup B_{2}$. Hence by induction, $A_{i} \geqq B_{j}$, for $j=1$ and 2. Hence by (2.2), $A_{1} \cap A_{2} \geqq B_{j}$, for $j=1$ and 2 .

Lemma 2. (1) $A \equiv A_{1} \cup A_{2} \geqq B_{1} \cap B_{2} \equiv B$ implies one of the following:

(i) $A \geqq B_{i}$, for $i=1$ or 2 ,

(ii) $A_{j} \geqq B$, for $j=1$ or 2 .

(2) $p \geqq B_{1} \cap B_{2}$ implies $p \geqq B_{i}$, for $i=1$ or 2 .

(3) $A_{1} \cup A_{2} \geqq p$ implies $A_{j} \geqq p$ for $j=1$ or 2 .

Proof. (1), (2) and (3) simply list the possibilities afforded by Definition 2.

(2) In this paper $p$, subscripted or not, will always denote an element of the partially ordered set $P$.

( $)(\equiv)$ denotes logical identity. 
LemMa 3. The relation ( $\geqq)$ on the words on the $p_{i}$ is reflexive and transitive.

Proof. Part I. $A \geqq A$. The proof is by induction on $\lambda(A)$. For $\lambda(A)=1$, $A \equiv p_{i}$ and the result follows from that property for the set $P$. Assume therefore that the result is verified when $\lambda(A)<k$, let $\lambda(A)=k$.

Case 1. $A \equiv A_{1} \cup A_{2}$. By induction hypothesis $A_{1} \geqq A_{1}$. By (2.1), $A_{1} \cup A_{2}$ $\geqq A_{1}$. Similarly, $A_{1} \cup A_{2} \geqq A_{2}$. Thus by (2.3), $A_{1} \cup A_{2} \geqq A_{1} \cup A_{2}$.

Case 2. $A \equiv A_{1} \cap A_{2}$. This case is the dual of Case 1 .

PART II. $A \geqq B$ and $B \geqq C$ implies $A \geqq C$. The proof is by induction on $\lambda(A)+\lambda(B)+\lambda(C)=\lambda$. When $\lambda=3, A \equiv p_{i}, B \equiv p_{j}, C \equiv p_{k}$ and the result follows from that property of the set $P$. Assume therefore that the result is verified when $\lambda<k$ and that $\lambda=k$.

Case 1.1.1. $B \equiv p_{i}, A \equiv p_{j}, C \equiv C_{1} \cup C_{2}$. From $p_{i} \geqq C_{1} \cup C_{2}$ and Lemma 1, $p_{i} \geqq C_{1}$ and $C_{2}$. Then, by induction hypothesis, $p_{j} \geqq C_{1}$ and $C_{2}$, thus $p_{j} \geqq C_{1} \cup C_{2}$ by (2.3).

Case 1.1.2. $B \equiv p_{i}, A \equiv p_{j}, C \equiv C_{1} \cap C_{2}$. From $p_{i} \geqq C_{1} \cap C_{2}$ and Lemma 2, $p_{i} \geqq C_{1}$ or $C_{2}$. Then, by induction hypothesis, $p_{j} \geqq C_{1}$ or $C_{2}$, thus $p_{j} \geqq C_{1} \cap C_{2}$ by (2.4).

Case 1.2. $B \equiv p_{i}, A \equiv A_{1} \cup A_{2}$. From $A_{1} \cup A_{2} \geqq p_{i}$ and Lemma $2, A_{1}$ or $A_{2} \geqq p_{i}$. Then, by induction hypothesis, $A_{1}$ or $A_{2} \geqq C$, thus $A_{1} \cup A_{2} \geqq C$ by (2.1).

Case 1.3. $B \equiv p_{i}, A \equiv A_{1} \cap A_{2}$. From $A_{1} \cap A_{2} \geqq p_{i}$ and Lemma $1, A_{1}$ and $A_{2} \geqq p_{i}$. Then, by induction hypothesis, $A_{1}$ and $A_{2} \geqq C$, thus $A_{1} \cap A_{2} \geqq C$ by (2.2).

Cases 1.1.1, 1.1.2, 1.2, and 1.3 exhaust all possible forms with $B \equiv p_{i}$.

Case 2.1. $B \equiv B_{1} \cup B_{2}, C \equiv p_{i}$. From $A \geqq B_{1} \cup B_{2}$ and Lemma $1, A \geqq B_{1}$ and $B_{2}$. From $B_{1} \cup B_{2} \geqq p_{i}$ and Lemma $2, B_{1}$ or $B_{2} \geqq p_{i}$. Then, by induction hypothesis, $A \geqq p_{i}$.

Case 2.2. $B \equiv B_{1} \cup B_{2}, \quad C \equiv C_{1} \cup C_{2}$. From $B \geqq C_{1} \cup C_{2}$ and Lemma 1 , $B \geqq C_{1}$ and $C_{2}$. Then, by induction hypothesis, $A \geqq C_{1}$ and $C_{2}$, thus $A \geqq C_{1} \cup C_{2}$ by (2.3).

Case 2.3. $B \equiv B_{1} \cup B_{2}, C \equiv C_{1} \cap C_{2}$. From $B_{1} \cup B_{2} \geqq C_{1} \cap C_{2}$ and Lemma 2, $B \geqq C_{1}$ or $B \geqq C_{2}$ or $B_{1} \geqq C$ or $B_{2} \geqq C$. If either of the first two alternatives holds, by induction hypothesis, $A \geqq C_{1}$ or $C_{2}$, hence $A \geqq C_{1} \cap C_{2}$ by (2.4). If either of the last two alternatives holds, consider $A \geqq B_{1} \cup B_{2}$. From Lemma 1 , $A \geqq B_{1}$ and $B_{2}$. Hence whichever of the last two alternatives holds, the induction hypothesis yields $A \geqq C$.

Cases $2.1,2.2$ and 2.3 exhaust all possible forms with $B \equiv B_{1} \cup B_{2}$. The forms with $B \equiv B_{1} \cap B_{2}$ are duals of these cases.

Now the relation $(\geqq)$ on the words may be extended to a partial ordering in the usual $\left({ }^{4}\right)$ way.

(4) For example, see Birkhoff [1, p. 4]. 
Definition 3. Two words $A$ and $B$ are equal if $A \geqq B$ and $B \geqq A$, written $A=B$.

It should now be verified that this is an equivalence relation and all further work should be carried out on the resulting equivalence classes. The elements of the lattice $\mathrm{CF}(P)$ will be these equivalence classes and the elements can only be represented by a word in that equivalence class. However no confusion will result if the terms "word" and "element" are used interchangeably. The formal details are left to the reader.

THEOREM 1. The partially ordered set obtained from the set of words on a partially ordered set $P$ by Definitions 2 and 3 is a lattice in which $A \cup B$ and $A \cap B$ are the least upper and greatest lower bounds, respectively, of $A$ and $B$. This lattice will henceforth be denoted $C F(P)$.

Proof. $A \cup B \geqq A$ and $B$ by Definition 2. Let $C \geqq A$ and $B$. Then by Definition 2, $C \geqq A \cup B$. Hence $A \cup B$ is the least upper bound of $A$ and $B$. A dual proof shows that $A \cap B$ is their greatest lower bound.

Because the definition of equality depends only on $(\geqq)$ and because the criteria for $(\geqq)$ is recursive and all words have finite length it is easily seen that we have proved

TheOREM 2. The word problem, i.e. the problem of deciding in a finite number of steps whether two given words are equal, is solved in $C F(P)$.

Definition 4. A partially ordered set $P$ with elements $p_{i}$ is said to be embedded in a lattice $L$, if $L$ possesses a subset of elements $s_{i}$ such that $s_{i} \leftrightarrow p_{i}$ is a $1-1$ correspondence with the property that $p_{i} \leqq p_{j}$ implies $s_{i} \leqq s_{j}$. The embedding is called minimal if the sublattice generated by the $s_{i}$ is $L$.

Theorem 3. $P$ is minimally embedded in $C F(P)$ and moreover any lattice in which $P$ can be minimally embedded is a homomorphic image of $C F(P)$.

Proof. The first part of the theorem follows immediately from the construction of $\mathrm{CF}(P)$. To prove the second part, let $L$ be any lattice in which $P$ is embedded. Let $P$ have elements $p_{i}$, the corresponding subset of $L$, elements $s_{i}$, and let $p_{i} \leftrightarrow s_{i}$ be the required correspondence. By assumption $S=\left\{s_{i}\right\}$ generates $L$. The correspondence $p_{i} \leftrightarrow s_{i}$ induces a natural correspondence between words on the $p_{i}$ and words on the $s_{i}$, hence a correspondence between the elements of $\mathrm{CF}(P)$ and $L$. If $f\left(x_{1}, \cdots, x_{n}\right)$ denotes a lattice polynomial or word on indeterminates $x_{1}, \cdots, x_{n}$, the mapping described is

$$
f\left(p_{i_{1}}, \cdots, p_{i_{n}}\right) \leftrightarrow f\left(s_{i_{1}}, \cdots, s_{i_{n}}\right)
$$

or simply

$$
f(P) \leftrightarrow f(S)
$$


for any substitution $p_{i_{1}}, \cdots, p_{i_{n}}$ for $x_{1}, \cdots, x_{n}$. Now $f\left(s_{i_{1}}, \cdots, s_{i_{n}}\right)$ is some element $a$ of $L$. It will be shown that the mapping

$$
f\left(p_{i_{1}}, \cdots, p_{i_{n}}\right) \rightarrow a
$$

obtained in this way is a homomorphism of $\mathrm{CF}(P)$ onto $L$. Clearly every word in $\mathrm{CF}(P)$ has an image in $L$ and since $S$ generates $L$, every element in $L$ is expressible as a word on the $s_{i}$, hence has a mate in $\operatorname{CF}(P)$.

It will be shown first that the mapping $(\mathrm{M})$ preserves the order relation $(\geqq)$ on the elements of $\mathrm{CF}(P)$. That is, if $f(P)$ and $g(P)$ are two words, and $f(P) \geqq g(P)$ in $\mathrm{CF}(P)$, then $f(S) \geqq g(S)$ in $L$. The proof is by induction on $\lambda[f(P)]+\lambda[g(P)]=\lambda$. When $\lambda=2, f(P) \equiv p_{i}, g(P) \equiv p_{j}$ and $p_{i} \geqq p_{j}$ implies $s_{i} \geqq s_{j}$ by the embedding property. Assuming the result for $\lambda<k$, let $\lambda=k$.

Case 1. $f(P) \equiv f_{1}(P) \cap f_{2}(P)$. By Lemma $1, f_{1}(P)$ and $f_{2}(P) \geqq g(P)$. Hence by induction hypothesis $f_{1}(S)$ and $f_{2}(S) \geqq g(S)$. Since $f_{1}(S), f_{2}(S)$ and $g(S)$ are elements of a lattice $L, f_{1}(S) \cap f_{2}(S) \geqq g(S)$. But $f(P) \rightarrow f(S) \equiv f_{1}(S) \cap f_{2}(S)$. The case $g(P)=g_{1}(P) \cup g_{2}(P)$ is the dual of Case 1 .

Case 2. $f(P) \equiv p_{i}$ and $g(P)=g_{1}(P) \cap g_{2}(P)$. From Lemma $2, p_{i} \geqq g_{1}(P)$ or $g_{2}(P)$. Hence, by induction hypothesis, $s_{i} \geqq g_{1}(S)$ or $g_{2}(S)$ and thus $s_{i} \geqq g_{1}(S)$ $\cap g_{2}(S)$. But $g(P) \rightarrow g(S) \equiv g_{1}(S) \cap g_{2}(S)$. Thus $s_{i} \geqq g(S)$. The case $g(P) \equiv p_{i}$ and $f(P)=f_{1}(P) \cup f_{2}(P)$ is the dual of Case 2 .

Case 3. $f(P) \equiv f_{1}(P) \cup f_{2}(P)$ and $g(P) \equiv g_{1}(P) \cap g_{2}(P)$. By Lemma 2 four possibilities must be examined. Suppose $f(P) \geqq g_{1}(P)$. By induction hypothesis $f(S) \geqq g_{1}(S)$ and thus $f(S) \geqq g_{1}(S) \cap g_{2}(S)$. The other possibilities are handled similarly.

Since $(\mathrm{M})$ preserves the order in $\mathrm{CF}(P)$ it is clear that $(\mathrm{N})$ does also. Thus, equality in $\mathrm{CF}(P)$ implies equality in $L$, conversely distinct elements in $L$ cannot correspond to equal words in $\mathrm{CF}(P)$.

It will now be shown that the correspondences $(\mathrm{M})$ and $(\mathrm{N})$ preserve unions and intersections in $\mathrm{CF}(P)$. Let $f(P) \rightarrow f(S)=a, g(P) \rightarrow g(S)=b$ and $f(P) \cup g(P)=h(P) \rightarrow h(S)=c$. It is to be shown that $c=a \cup b$. In any event $f(P) \cup g(P) \rightarrow f(S) \cup g(S)=a \cup b$. Since $f(P) \cup g(P)=h(P)$, the preceding paragraph shows that $h(S)=a \cup b$. Similarly intersections are preserved.

TheOREM 4. $F L(P)$ and $C F(P)$ are identical if and only if $P$ has the following two properties:

(i) $p_{i}=l . u . b .\left(p_{j}, p_{k}\right)$ if and only if $i=j$ or $k$.

(ii) $p_{i}=$ g.l.b. $\left(p_{j}, p_{k}\right)$ if and only if $i=j$ or $k$.

Proof. Necessity. Suppose that $\mathrm{FL}(P)$ and $\mathrm{CF}(P)$ are identical and that $p_{i}=$ l.u.b. $\left(p_{j}, p_{k}\right)$. Then $p_{i}=p_{j} \cup_{p_{k}}$ in $\mathrm{FL}(P)$, since it is to preserve all existing bounds of pairs of elements. But in $\mathrm{CF}(P), p_{i}=p_{j} \cup p_{k}$ implies $p_{j} \cup p_{k} \geqq p_{i}$, hence, $p_{j} \geqq p_{i}$ or $p_{k} \geqq p_{i}$, while on the other hand, $p_{i} \geqq p_{j}$ and $p_{k}$. Thus $p_{i}=p_{j}$ or $p_{k}$, i.e. $i=j$ or $k$. (ii) is established in a dual way.

Sufficiency. Since the elements of $\mathrm{FL}(P)$ and $\mathrm{CF}(P)$ are represented by 
the same set of words it suffices to prove that equality of words in $\mathrm{FL}(P)$ is equivalent to equality in $\mathrm{CF}(P)$. Since $\mathrm{FL}(P)$ provides a minimal embedding for $P$, by Theorem $3, \mathrm{CF}(P) \rightarrow \mathrm{FL}(P)$, hence equality in $\mathrm{CF}(P)$ implies equality in $\mathrm{FL}(P)$.

The converse is established by showing that for words $A, B, A \geqq B$ in $\mathrm{FL}(P)$ implies $A \geqq B$ in $\mathrm{CF}(P)$. The notation used in this proof follows Dilworth's $[2$, p. 126].

Lemma 4. If $v(A)$ exists, i.e. $A=v(A)=p_{i}$, for some $i$, in $F L(P), A=p_{i}$ in $C F(P)$.

Proof. The proof is by induction on $\lambda(A)$. When $\lambda(A)=1, A \equiv p_{i}$ and $v(A)=p_{i}$ in $\mathrm{FL}(P)$, hence $A=p_{i}$ in $\mathrm{CF}(P)$. If $A \equiv A_{1} \cup A_{2}$ and $v(A)$ exists, then $v\left(A_{1}\right), v\left(A_{2}\right)$ exist and l.u.b. $\left[v\left(A_{1}\right), v\left(A_{2}\right)\right]$ exists in $P$ and equals $v(A)$. Let $v\left(A_{1}\right)=p_{j}, v\left(A_{2}\right)=p_{k}$. Then l.u.b. $\left(p_{j}, p_{k}\right)=p_{j}$ or $p_{k}$, from the assumption (i) on $P$. Without loss of generality, let $p_{j}=1$.u.b. $\left(p_{j}, p_{k}\right)$ so that $p_{j} \geqq p_{k}$. Thus in $\operatorname{CF}(P)$, by induction, $A_{1}=p_{j}, A_{2}=p_{k}$ and thus in $\mathrm{CF}(P), A_{1} \cup A_{2}$ $=p_{j} \cup p_{k}=p_{j}$. Thus $A=p_{j}$ in $\mathrm{CF}(P)$ as was to be proved. A dual proof handles the other case when $A \equiv A_{1} \cap A_{2}$.

Now suppose $A \geqq B$ in $\mathrm{FL}(P)$. By virtue of (iii) in Dilworth's definition (1.5) $A \geqq B(n)$. Proceeding now by induction on $n$, let $A \geqq B(1)$. If $A \equiv B$ there is nothing to prove. If $v(A), v(B)$ exist and $v(A) \geqq v(B)$ in $P$, then in $\mathrm{CF}(P)$, by Lemma $4, A=v(A), B=v(B)$, then $A \geqq v(A) \geqq v(B) \geqq B$ in CF $(P)$. If $A \geqq B(n)$ one of (1)-(5) must hold. Induction hypothesis gives the corresponding result in $\mathrm{CF}(P)$, and either the transitivity of $\geqq$ or one of (2.1)(2.4) in our Definition 2 implies $A \geqq B$ in $\mathrm{CF}(P)$.

In what follows it is convenient to consider expressions of the form $A_{1} \cup \ldots \cup A_{m}, m$ finite. While such expressions are not words as defined they shall be used here to represent a word of the form

$$
\left(\cdots\left(A_{1} \cup A_{2}\right) \cup \cdots \cup A_{m-1}\right) \cup A_{m}
$$

or any word obtained from this form by use of the associative and commutative laws. In the sequel $(\equiv)$ shall be used to denote not only logically equivalent words, but also words which become logically equivalent when appropriately operated upon by the associative and commutative laws alone, writing $A_{1} \cup \ldots \cup A_{n}$ for any one such word, more frequently $\cup_{i} A_{i}$, tacitly assuming that only a finite number of $A_{i}$ are involved.

Lemma 1 and (1) of Lemma 2 then may be extended by an easy induction to any finite number of terms, rather than just 2. Much use will be made of these two lemmas and special reference to them will be omitted.

\section{Canonical forms and sublattices in $\mathrm{CF}(P)$.}

Theorem 5. Let $P$ be any partially ordered set. In $C F(P)$, if $A \equiv \mathrm{U}_{i} A_{i}$, $A_{i} \equiv \bigcap_{j} a_{i j}$ or $A_{i} \equiv p$, an element of $P$, then there is a shorter word $B$ equal to $A$ if and only if one or more of the following hold: 
(1) For some $i, A_{i}$ is equal to a shorter word, $A_{i}^{\prime}$.

(2) $A_{k} \geqq A_{k}$ for some $h$ and $k, h \neq k$.

(3) $A \equiv \bigcup_{i} A_{i} \geqq a_{h k}$ for some $h$ and $k$ where $A_{h} \not \equiv p$.

Proof. (1) and (2) are clearly sufficient. To see that (3) is sufficient consider $B \equiv \bigcup_{i \neq h} A_{i} \cup a_{h k} . B$ has shorter length than $A$ since $\lambda\left(a_{h k}\right)<\lambda\left(A_{h}\right)$ as $A_{h} \neq \equiv$. $A \geqq B$ since $A \geqq a_{h k}$ by hypothesis, and $A \geqq \bigcup_{i \neq h} A_{i}$. Conversely $B \geqq A$ since $a_{h k} \geqq \bigcap_{j} a_{h j} \equiv A_{h}$ and therefore $\bigcup_{i \neq h} A_{i} \cup a_{h k} \geqq \bigcup_{i \neq h} A_{i} \cup A_{h} \equiv A$. Thus $A=B$.

To prove the necessity, take $B$ to be the shortest (and shorter than $A$ ) word equal to $A$.

Case 1 . $B=p$. Thus $p=\cup_{i} A_{i}$, hence $p \geqq \bigcup_{i} A_{i}$ and $p \geqq A_{i}$ for all $i$. Conversely $\cup_{i} A_{i} \geqq p$ and hence $A_{h} \geqq p$ for some $h$. Thus $A_{h} \geqq p \geqq A_{i}$ for some $h$ and all $i$, in particular condition (2) is satisfied.

Case 2. $B \equiv \bigcap_{i} B_{i}$. From $B \geqq A$ it follows that $B_{i} \geqq A_{j}$ for all $i$ and $j$. From $A \geqq B$ it follows that either $A \geqq B_{h}$ for some $h$ or $A_{k}>B$ for some $k$. When the first alternative holds, $\bigcap_{i} B_{i} \equiv B \geqq A \geqq B_{h} \geqq \bigcap_{i} B_{i}$, thus $A=B=B_{h}$, contrary to the choice of $B$ as the shortest word equal to $A$. When the second alternative holds, $A \geqq A_{k} \geqq B \geqq A$. Hence $A=A_{k}$ and $A_{k} \geqq A_{i}$, for all $i$, in particular condition (2) is satisfied.

Case 3. $B \equiv \mathrm{U}_{r} B_{r}$. It may be assumed $B_{r} \equiv \bigcap_{s} b_{r s}$ or $B_{r} \equiv p$. From $A \geqq B$ it follows that $\bigcup_{i} A_{i} \geqq B_{r}=\bigcap_{s} b_{r s}$ for all $r$. Thus either: (i) for all $r$, there exists an index $j(r)$ such that $A_{j(r)} \geqq B_{r}$, or (ii) the first alternative does not hold and for some $r$ and $t, \cup_{i} A_{i} \geqq b_{r t}$ and $\lambda\left(b_{r t}\right)<\lambda\left(B_{r}\right)$. (For if the lengths were equal then $B_{r} \equiv b_{r t} \equiv p$ and for this $r$, there would have to be a $j(r)$ such that $A_{j(r)} \geqq B_{r}$ $\equiv p$.) Similarly from $B \geqq A, \cup_{r} B_{r} \geqq A_{i} \equiv \bigcap_{j} a_{i j}$ for all $i$, two alternatives are possible: (iii) for all $i$, there exists an index $k(i)$ such that $B_{k(i)} \geqq A_{i}$ or (iv) the third alternative does not hold, and for some $i$ and $h, U_{r} B_{r} \geqq a_{i h}$ and $\lambda\left(a_{i h}\right)<\lambda\left(A_{i}\right)$.

Suppose that alternative (ii) holds. Thus $\cup_{i} B_{i} \geqq b_{r t}$ and, as in the proof of the sufficiency of (3), $\bigcup_{i} B_{i}=\bigcup_{i \neq r} B_{i} \cup b_{r t}$, contrary to the choice of $B$ as the shortest word equal to $A$. Suppose that alternative (iv) holds. Thus $\cup_{j} A_{j} \geqq a_{i h}$, which is condition (3).

Thus it may be assumed that (i) and (iii) hold simultaneously. Hence, combining, $B_{r} \leqq A_{j(r)} \leqq B_{k[j(r)]}$ for all $r$. If, for some $r, r \neq k[j(r)]=t$, then $B_{r} \leqq B_{t}, r \neq t$ and $B \equiv \bigcup_{s} B_{s}=\bigcup_{s \neq r} B_{s}$, contrary to the choice of $B$ as the shortest word equal to $A$. Hence assume that $r=k[j(r)]$ for all $r$.

Now suppose that, for some $r$, there exist distinct indices $j_{1}(r)$ and $j_{2}(r)$ such that $A_{j_{1}(r)} \geqq B_{r}$ and $A_{j_{2}(r)} \geqq B_{r}$. Then from (3)

$$
\begin{aligned}
& B_{k\left[j_{1}(r)\right]} \geqq A_{j_{1}(r)} \geqq B_{r}, \\
& B_{k\left[j_{2}(r)\right]} \geqq A_{j_{2}(r)} \geqq B_{r} .
\end{aligned}
$$

But $k\left[j_{1}(r)\right]=r=k\left[j_{2}(r)\right]$ as was just shown. Hence $A_{j_{2}(r)}=A_{j_{1}(r)}$ and in particular, condition (2) holds. Thus without loss of generality suppose in (i) that for every $r$ there is a unique index $j(r)$. 
Similarly, $A_{i} \leqq B_{k(i)} \leqq A_{j[k(i)]}$. If, for some $i, i \neq j[k(i)]=h$, then $A_{i} \leqq A_{h}$ and condition (2) holds. Hence assume $i=j[k(i)]$ for all $i$. If there are distinct indices $k_{1}(i)$ and $k_{2}(i)$ such that $B_{k_{1}(i)} \geqq A_{i}$ and $B_{k_{2}(i)} \geqq A_{i}$, then from (1)

$$
\begin{aligned}
& A_{j\left[k_{1}(i)\right]} \geqq B_{k_{1}(i)} \geqq A_{i}, \\
& A_{j\left[k_{2}(i)\right]} \geqq B_{k_{2}(i)} \geqq A_{i} .
\end{aligned}
$$

But $i=j\left[k_{1}(i)\right]=j\left[k_{2}(i)\right]$ as was just shown, hence $B_{k_{1}(i)}=B_{k_{2}(i)}$ and therefore $B \equiv \bigcup_{r}, B_{r}=\bigcup_{r \neq k_{1}(i)} B_{r}$, contradicting the choice of $B$ as the shortest word equal to $A$. Hence, in (iii), for every $i$, there exists a unique index $k(i)$. Moreover from

$$
A_{i}=A_{j[k(i)]} \geqq B_{k(i)} \geqq A_{i} ; B_{r}=B_{k[j(r)]} \geqq A_{j(r)} \geqq B_{r}
$$

it follows that $A_{i}=B_{k(i)}$ and $B_{r}=A_{j(r)}$, and $i \leftrightarrow k(i)$ is a 1-1 correspondence between the $A_{i}$ 's and $B_{r}$ 's. But since $\lambda(A)>\lambda(B)$ it follows that for some $i$, $\lambda\left(A_{i}\right)>\lambda\left(B_{k(i)}\right)$, which is condition (1).

The dual theorem is of course valid.

Corollary 1. If $A=B$ then either $A \equiv B$ or there exists a $C$ such that $A=B=C$ and $\lambda(C)<\lambda(A)$ or $\lambda(B)$.

Proof. It may be supposed that $\lambda(A)=\lambda(B)$, for if not, take $C$ to be the shorter word. The proof is by induction on $\lambda(A)=\lambda(B)=\lambda$. When $\lambda=1$, $A=B$ implies $A \equiv B \equiv p$. Assuming the corollary when $\lambda<k$, let $\lambda=k$. Taking $B$ as in the theorem, it is easily seen that a word shorter than $A$ or shorter than $B$ is produced, except perhaps in the last case where it might occur that $\lambda(A)=\lambda(B)$ and $A_{i}=B_{k(i)}$ all $(i)$. But then, by induction either a shorter word $C_{i}$ exists or by application of the commutative and associative laws $A_{i}$ may be derived from $B_{k(i)}$. If the latter alternative holds for all $i, A$ can be derived from $B$ by application of the associative and commutative laws alone. If the former alternative holds for one index $i$, replacing $A_{i}$ or $B_{k(i)}$ by $C_{i}$ gives a word shorter, but equal to $A$ and $B$.

Definition 5. A word $W$ is canonical if $A=W$ implies $\lambda(A) \geqq \lambda(W)$.

COROLlaRY 2. If $\cup_{j} A_{j}=\cup_{i} B_{i}$ and $\cup_{i} B_{i}$ is canonical, then for every $i$ there exists a $j$ such that $B_{i} \leqq A_{j}$.

Proof. If $B_{i} \equiv p$, then the result follows from the extended form of Lemma 2. If $B_{i} \equiv \bigcap_{r} b_{i r}$, consider $\cup_{j} A_{j} \geqq B_{i} \equiv \bigcap_{r} b_{i r}$. Either $A_{j} \geqq B_{i}$ for some $j$ or $\cup_{j} A_{j} \geqq b_{i r}$ for some $i$. The first case is the desired conclusion. The second yields the condition $\cup_{i} B_{i} \geqq b_{i r}$, which, by Theorem 5 , is sufficient to construct a word shorter than $U_{i} B_{i}$ and equal to it.

Corollary 3. If $A=\cup_{i} A_{i}=\bigcap_{j} B_{j}=B$ and $\bigcap_{j} B_{j}$ is canonical, then $\mathrm{U}_{i} A_{i}=A_{h}$ for some $h$.

Proof. For all $i$ and $j, B_{j} \geqq A_{i}$ as $B \geqq A$. From $A \geqq B, \cup_{i} A_{i} \geqq \bigcap_{j} B_{j}$. From 
Lemma 2 , either $A \geqq B_{j}$, for some $j$, or $A_{h} \geqq B$. If the first case holds, $B \geqq A$ $\geqq B_{j} \geqq B$ and $B=B_{j}$, but $B$ was assumed canonical. Therefore $A \geqq A_{h} \geqq B \geqq A$ and $A \equiv \mathrm{U}_{i} A_{i}=A_{h}$.

Dual corollaries hold of course.

Lemma 5. Let $P$ be a partially ordered set with elements $p_{i}$. In $C F(P)$, for any index $j$ and for any word $A$,

(1) $p_{j} \leqq A$ or

(2) there exists a finite subset of indices $R$ such that $R$ has no indices in common with $S_{j}=\left[k \mid p_{j} \leqq p_{k}\right.$ in $\left.P\right]$ and $A \leqq \bigcup_{i \in R} p_{i}$.

(1) and (2) are mutually exclusive.

Proof. To show that (1) and (2) must be mutually exclusive let $R$ be any subset of indices disjoint from $S_{j}$ and suppose that (1) and (2) both hold. Thus $p_{j} \leqq U_{i \in R} p_{i}$, which implies that there exists an index $i \in R$ such that $p_{j} \leqq p_{i}$. But then $i \in S_{j}$, contrary to the supposed disjointness of $R$ and $S_{j}$.

That (1) or (2) must hold is proved by induction on $\lambda(A)$. When $\lambda(A)=1$, $A \equiv p_{k}$. Now either $p_{j} \leqq p_{k}$ or $p_{j} \$ p_{k}$. In the second case let $R$ consist of $k$ alone and (2) holds trivially. When $A \equiv A_{1} \cup A_{2}$ and $p_{j}$ and $p_{j} A_{2}$. Hence by induction there exist finite index sets $R_{1}$ and $R_{2}$ each disjoint from $S_{j}$ such that $A_{1} \leqq \bigcup_{i \in R_{1}} p_{i}$ and $A_{2} \leqq \bigcup_{i \in R_{2}} p_{i}$. Let $R$ be the (finite) set composed of the indices in $R_{1}$ and $R_{2}$. Clearly $R$ is disjoint from $S_{j}$ and $A_{1} \leqq \bigcup_{i \in R_{1}} p_{i} \leqq \bigcup_{i \in R} p_{i}$ while $A_{2} \leqq \bigcup_{i \in R_{2}} p_{i} \leqq \bigcup_{i \in R} p_{i}$. Thus $A_{1} \cup A_{2}$ $\leqq \bigcup_{i \in R} p_{i}$. When $A \equiv A_{1} \cap A_{2}$ and $p_{j} \leq A_{1} \cap A_{2}$ then $p_{j} \leq A_{h}, h=1$ or 2 . Then by induction there exists a finite index set $R$ disjoint from $S_{j}$ such that $A_{h} \leqq \bigcup_{i \in R} p_{i}$ and so $A_{1} \cap A_{2} \leqq \bigcup_{i \in R} p_{i}$. A dual lemma holds of course. When $P$ is a finite set, the subset $R$ may always be chosen to be all subscripts not in $S_{j}$.

THEOREM 6. Let $T=\left\{t_{i}\right\}$ be any subset of $C F(P)$ with the property that, for any finite subset of indices $R, t_{j} \leqq \bigcup_{i \in R} t_{i}$ implies that there exists $k \in R$ such that $t_{j} \leqq t_{k}$ in $T$, and the dual property. Then the sublattice $L(T)$ of $C F(P)$ generated by $T$ is lattice isomorphic to $C F(Q)$ where $Q$ is a partially ordered set order isomorphic $\left({ }^{5}\right)$ to $T$.

Proof. Let the elements of $Q$ be denoted by $q_{i}$ and the correspondence $q_{i} \leftrightarrow t_{i}$. It is clear that $L(T)$ provdes a minimal embedding for $Q$; hence $L(T)$ is a homomorphic image of $\mathrm{CF}(Q)$ through the mapping generated by $q_{i} \rightarrow t_{i}$. To show that this is indeed one to one it only remains to be shown that equality of words in $L(T)$ implies the equality of the corresponding words in $\mathrm{CF}(Q)$. Using the notation for words employed in Theorem 3 , it suffices to show that for two words $f(T)$ and $g(T), f(T) \leqq g(T)$ in $L(T)$ implies $f(Q) \leqq g(Q)$ in $\mathrm{CF}(Q)$. The proof is by induction on $\lambda[f(Q)]+\lambda[g(Q)]=\lambda$.

(s) The elements of $Q$ and $T$ can be placed in a 1-1 correspondence so that order is preserved in both directions. 
When $\lambda=2, f(T) \equiv t_{i}, g(T) \equiv t_{j}$ and $t_{i} \leqq t_{j}$ implies $q_{i} \leqq q_{j}$ because $T$ and $Q$ are assumed isomorphic. Assuming the result when $\lambda<k$, let $\lambda=k$.

Case 1. $f(T) \equiv t_{j}$. From Lemma 5 , in $\mathrm{CF}(Q)$, either $q_{j} \leqq g(Q)$ or $g(Q)$ $\leqq U_{i \in R} q_{i}$, for some finite set of indices $R$, disjoint from $S_{j}$. If the latter alternative holds then $g(T) \leqq \bigcup_{i \in R} t_{i}$ in $L(T)$, since $L(T)$ is a lattice homomorphic image of $\mathrm{CF}(Q)$. This, together with the assumption $t_{j} \leqq g(T)$ and the hypothesis of the theorem implies that $t_{j} \leqq t_{i}$ for some $i \in R$ contrary to the condition that $R$ be disjoint from $S_{j}$. Hence the first alternative must hold, as was to be shown. The case $g(T) \equiv t_{j}$ is the dual of Case 1 .

Case 2. $f(T) \doteq f_{1}(T) \cup f_{2}(T) \leqq g(T)$. Since $L(T)$ is a lattice $f_{1}(T)$ and $f_{2}(T) \leqq g(T)$. Then by induction, $f_{1}(Q)$ and $f_{2}(Q) \leqq g(Q)$, hence $f(Q) \equiv f_{1}(Q)$ $\cup f_{2}(Q) \leqq g(Q)$. The case $g(T) \equiv g_{1}(T) \cap g_{2}(T)$ is the dual of Case 2 .

Case 3. $f(T) \equiv f_{1}(T) \cap f_{2}(T) \leqq g_{1}(T) \cup g_{2}(T) \equiv g(T)$. Since $L(T)$ is a sublattice of $\mathrm{CF}(P)$ the words $f, f_{1}, f_{2}, g_{1}, g_{2}$ and $g$, up to now regarded as words on the $t_{i}$, may also be regarded as words on the $p_{i}$. Rewriting the condition of Case $3, f(P) \equiv f_{1}(P) \cap f_{2}(P) \leqq g_{1}(P) \cup g_{2}(P) \equiv g(P)$. By Lemma 2, either $f(P)$ $\leqq g_{i}(P), i=1$ or 2 , or $f_{j}(P) \leqq g(P), j=1$ or 2 . These words may again be considered as words on the $t_{i}, f(T) \leqq g_{1}(T), i=1$ or 2 and $f_{j}(T) \leqq g(T), j=1$ or 2 , and applying the induction hypotheses to whichever condition holds, obtain $f(P) \leqq g(P)$.

The conditions of Theorem 6 are clearly necessary for $L(T)$ to be isomorphic to $\mathrm{CF}(Q)$, for in $\mathrm{CF}(Q), q_{j} \leqq \mathrm{U}_{i \in R} q_{i}$ implies $q_{j} \leqq q_{k}$, for some $k \in R$, and dually.

This section is concluded with a theorem giving sufficient conditions for a sublattice of $\mathrm{CF}(P)$ to be isomorphic to $\mathrm{FL}(P)$. The proof will require Lemma 5 stated for $\mathrm{FL}(P)$. This follows by the homomorphism $\mathrm{CF}(P)$ $\rightarrow \mathrm{FL}(P)$; however it is no longer true that (1) and (2) are mutually exclusive. The following lemma is also needed.

LEMMA 6. In $F L(P)$, for any subscript $i$ and any word $A$ either

(1) $p_{i} \leqq A$ or

(2) there exists a finite set $V$ of pairs of indices $(m, n)$ such that $V$ has no pair in common with

$$
T_{i}=\left[(j, k) \mid l . u . b .\left(p_{j}, p_{k}\right)=p_{h} \text { in } P, p_{i} \leqq p_{h} \text { in } P\right]
$$

and $A \leqq \mathrm{U}_{(m, n) \in V}\left(p_{m} \cup_{p_{n}}\right)$.

Proof. If $p_{i} \neq p_{h}$, then $(h, h) \notin T_{i}$. Thus if $p_{i} \ddagger A$, for the subset $V$ use the indices of the finite set $R$ given by Lemma 5 and take $V=[(h, h) \mid h \in R]$. Thus $V$ and $T_{i}$ are disjoint and $\bigcup_{h \in R} p_{h}=U_{(h, h) \in V}\left(p_{h} \cup_{p_{h}}\right)=U_{(j, k) \in V}\left(p_{j} \cup_{p_{k}}\right)$, and the result follows from Lemma 5 , restated for $\operatorname{FL}(P)$.

Theorem 7. Let $P$ be a partially ordered set of elements $p_{i}$. In $C F(P)$ let there exist a subset $S$ of elements $s_{j}$ such that $p_{i} \leftrightarrow s_{i}$ is a 1-1 correspondence and

(1) $s_{k}=s_{i} \cup s_{j}$ if and only if $p_{k}=l . u . b .\left(p_{i}, p_{j}\right)$, and dually, and 
(2) $s_{i} \leqq \bigcup_{(j, k) \in T}\left(s_{j} \cup s_{k}\right)$, where $T$ is any finite collection of pairs of indices implies for some pair $(j, k)$ in $T$ and some $h, s_{h}=s_{j} \cup s_{k}$ and $s_{i} \leqq s_{h}$ in $C F(P)$, and dually.

Then the sublattice $L(S)$ of $C F(P)$ generated by $S$ is lattice isomorphic to $F L(P)$.

Proof. Clearly $P$ is embedded in $L(S)$ and by condition (1), $L(S)$ preserves least upper and greatest lower bounds existing in $P$; hence $p_{i} \rightarrow s_{i}$ generates a homomorphism of $\mathrm{FL}(P)$ into $L(S)$, and equality of words on the $p_{i}$ in $\mathrm{FL}(P)$ implies equality of the corresponding words on the $s_{i}$. To prove the converse it suffices to show, using the notation of Theorem 3 , that $f(S) \leqq g(S)$ in $L(S)$ implies $f(P) \leqq g(P)$ in $\mathrm{FL}(P)$. The proof is by induction on $\lambda[f(P)]$ $+\lambda[g(P)]=\lambda$. For $\lambda=2, f(P) \equiv s_{i}, g(P) \equiv s_{j}$ and the result follows from condition (1). Assume the result for $\lambda<k$ and let $\lambda=k$.

Case 1. $f(S) \equiv s_{i} \leqq g(S)$. Consider $p_{i}$ and $g(P)$. By Lemma $6, p_{i} \leqq g(P)$ or $g(P) \leqq \bigcup_{(j, k) \in V}\left(p_{j} \cup_{p_{k}}\right)$ for some finite subset $V$ disjoint from $T_{i}$. If the latter condition holds then $g(S) \leqq \bigcup_{(j, k) \in V}\left(s_{j} \cup s_{k}\right)$ where $V$ is disjoint from $T_{i}^{\prime}$, where now

$$
T_{i}^{\prime}=\left[(m, n) / \exists t \ni: s_{t}=s_{m} \cup s_{n} \text { and } s_{i} \leqq s_{t} \text { in } L(S)\right],
$$

by condition (1) and the definition of $T_{i}$. This, together with the assumption $s_{i} \leqq g(S)$ and the hypothesis of the theorem, implies that there is a pair $(j, k) \in V$ such that $s_{h}=s_{j} \cup s_{k}$ and $s_{i} \leqq s_{h}$, or that $(j, k) \in T_{i}^{\prime}$, contrary to the condition that $V$ and $T_{i}^{\prime}$ are disjoint. Hence the first condition must hold, as was to be shown. The case $f(S) \leqq g(S) \equiv s_{i}$ is the dual.

The remainder of the proof is analogous to that given for Cases 2 and 3 in the proof of Theorem 6.

4. Examples. Consider the partially ordered set, $P$, consisting of four elements, $t, u, v$, and $w$, with $t>u$ and $v>w$ as its only order relations. By Theorem 4, $\mathrm{CF}(P)$ is identical with $\mathrm{FL}(P)$. Four infinite descending chains in $\mathrm{FL}(P)$ will now be constructed. Let $A_{1} \equiv t, B_{1} \equiv t \cup_{w}, C_{1} \equiv(t \cup w) \cap v$, $D_{1} \equiv[(t \cup w) \cap v] \cup u$ and for $n \geqq 2, A_{n} \equiv D_{n-1} \cap t, B_{n} \equiv A_{n} \cup w, C_{n} \equiv B_{n} \cap v$, $D_{n} \equiv C_{n} \cup u$. Clearly $A_{1} \geqq A_{2} \geqq \cdots \geqq A_{n} \geqq \cdots ; B_{1} \geqq B_{2} \geqq \cdots \geqq B_{n} \geqq \cdots$; $C_{1} \geqq C_{2} \geqq \cdots \geqq C_{n} \geqq \cdots ; D_{1} \geqq D_{2} \geqq \cdots \geqq D_{n} \geqq \cdots$. The following lemma shows that all these containing relations are proper.

Lemma 7. $A_{n}, B_{n}, C_{n}, D_{n}$ are in canonical form, for all $n$.

Thus, since the lengths of the words are strictly increasing no equality could hold.

The proof of Lemma 7 is made by a straightforward induction on $n$, using Theorem 5 and its dual to show, in turn, that $A_{n}, B_{n}, C_{n}$, and $D_{n}$ are in canonical form.

Consider the partially ordered set, $Q$, consisting of five elements, $a, b, c, d$, and $e$ with $a>b>c>d$ as its only order relations. By Theorem $3, \mathrm{CF}(Q)$ is 
identical with $\mathrm{FL}(Q)$. Consider the elements $T \equiv b, U \equiv c, V \equiv e \cup d$ and $W \equiv a \cap e$. The sublattice they generate is isomorphic to $\operatorname{CF}(P)$, where $P$ is the set described above. This is proved by verifying the criteria of Theorem 6 . It is easy to see that the set $T, U, V, W$ is order isomorphic with $P$. The other criteria then reduce to verifying $b \leq c \cup(e \cup d)$ and its dual which can be done by inspection.

\section{BIBLIOGRAPHY}

1. G. Birkhoff, Lattice theory, rev. ed., Amer. Math. Soc. Colloquium Publications, vol. 25, New York, 1948.

2. R. P. Dilworth, Lattices with unique complements, Trans. Amer. Math. Soc. vol. 57 (1945) pp. 123-154.

3. T. Evans, The word problem for abstract algebras, J. London Math. Soc. vol. 26 (1951) pp. 64-71.

4. Yu. I. Sorkin, Free unions of lattices, Rec. Math. (Mat. Sbornik) N.S. (72) vol. 30 (1952) pp. 677-694 (Russian).

5. P. M. Whitman, Free lattices, I. Ann. of Math. (2) vol. 42 (1941) pp. 325-330.

6. - Free lattices, II. Ann. of Math. (2) vol. 43 (1942) pp. 104-105.

California institute of Technology, Pasadena, Calif. 